\title{
From nuclear astrophysics to fundamental nuclear physics: challenging experimental approaches at $\mathbf{n}$ TOF (CERN)
}

\author{
Agatino Musumarra ${ }^{1,2}$ for the n_TOF collaboration \\ ${ }^{1}$ Dipartimento di Fisica e Astronomia, DFA University of Catania 95123 Catania, Italy \\ ${ }^{2}$ Istituto Nazionale di Fisica Nucleare, INFN Sezione di Catania 95123 Catania, Italy
}

\begin{abstract}
The n_TOF installation at CERN is one of the leading neutron facilities worldwide undergoing a major update of the neutron spallation source. The update will provide improved n-TOF resolution in the experimental areas and the possibility to perform neutron cross section measurements at very high neutron flux (NEAR-Station). The renewed capabilities of the facility must be supported by smart and nonconventional experimental approaches. In this framework two examples will be reported. The first one concerns the measurement of a key reaction channel involved in Primordial Nucleosynthesis: the ${ }^{7} \operatorname{Be}(n, \alpha)$, by using a radioactive ${ }^{7} \mathrm{Be}$ target. The second one provides a state-of-the-art scenario for the $n-n$ scattering length measurement. This will be performed by neutron-deuteron $(n-d)$ breakup three-body reaction. In this case, the envisaged experimental setup will provide a complete three-body kinematic reconstruction. By these important physics cases we are crossing the technological frontiers for charged particle and neutron detection.
\end{abstract}

\section{The n_TOF facility}

The n_TOF facility at CERN [1], operating since 2001, provides a unique opportunity for measuring neutron cross sections of interest to fundamental nuclear physics, nuclear astrophysics and neutron induced reactions for advanced nuclear technologies. The qualifying features of the facility are the wide energy range that can be covered in a single measurement and the large yield of neutrons produced by spallation of a single proton pulse provided by the CERN Proton Synchrotron (PS). More in detail, the new, high-flux experimental area (EAR2) allows us to measure neutron cross section relevant for nuclear astrophysics covering the region of interest for Big-Bang, stellar and s-process nucleosynthesis. The main advantage of the EAR2 beam line, in operation since August 2014, with respect to the first experimental area (EAR1), is the high neutron flux, of $10^{8}$ neutrons/pulse (see fig. 1), delivered in a short time interval, which results in a convenient signal-tobackground ratio when measuring small mass and/or highly radioactive targets, as well as isotopes characterized by a small reaction cross-section [2].

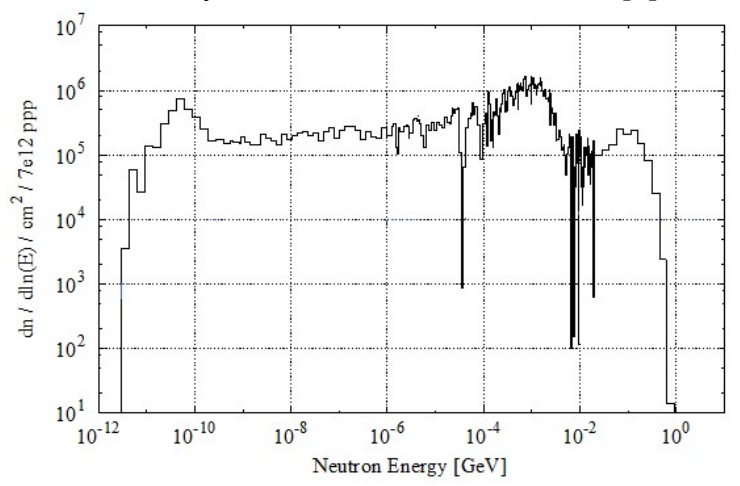

Fig. 1. $n$-fluence in EAR2.

Consequently, $\mathrm{n}$ TOF is one of the few time-of-flight facilities worldwide where neutron cross sections on radioactive isotopes of short half-life can be measured.
On the other hand, the facility has been complemented by a state-of-the-art detection systems and highperformance data acquisition systems. It follows that, apart for the neutron beam, another challenge in many measurements is the availability of a suitable amounts of radioactive isotopes to be used as target. For instance, the $n$ TOF collaboration involves a group working at Paul-Scherrer-Institute (PSI), Zurich, able to extract and separate relatively large amount of radioactive isotope for producing targets. This scientific synergy makes possible to measure on short lifetime isotopes (e.g., ${ }^{7} \mathrm{Be}$ ) in a very effective way, as shown in the following chapters.

After the full exploitation of the second-generation $\mathrm{n}$ TOF neutron spallation target, the facility underwent an important upgrade during the last CERN's Long Shutdown (restart in June 2021). The upgrade concerns the installation of a new spallation target. The first and second-generation lead targets were water-cooled, and they were designed for maximizing performances of the EAR1 beam line, while the new target is gas cooled, in order to avoid corrosion issues and contamination of the cooling system; the design has been also optimized for the vertical flight path of EAR2, providing an improved Time-of-Flight resolution at low beam energy.

In addition to the new spallation target, the installation of a new experimental area, close to the target and outside the shielding wall of the targetmoderator, has been implemented (fig.2). The new experimental site, the NEAR station (LoI CERN-INTC2020-073; INTC-I-2223), will take advantage of the extremely high neutron fluence expected at a position close to the spallation target. The dramatic increase in neutron fluence will make possible activation measurements on relatively small-mass samples and on radioactive isotopes. In this case the neutron beam will be transported to the NEAR Station through a small aperture in the shielding wall, traversing a suitable collimator/moderator system. As a matter of fact, the NEAR Station will operate in parallel with the two existing beam lines (EAR1 and EAR2), improving the 
overall facility capabilities. Monte Carlo simulations show that, as a result of a correct combination of the moderator material with the filter geometry, a Maxwellian-like neutron spectrum can be produced (from a few $\mathrm{keV}$ to a few tens of $\mathrm{keV}$ ). This low energy n-beam will allow measuring Maxwellian Average Cross Sections (MACS) by using activation techniques. It is worthwhile to mention that the possibility to experimentally determine MACS for short-lived unstable nuclei opens the possibility to investigate with high reliability the branching points in the s-process and weak r-process nucleosynthesis scenarios.

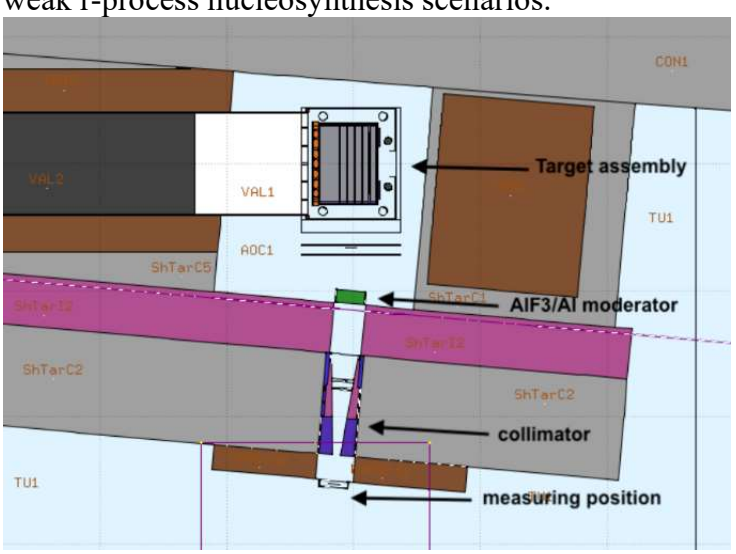

Fig. 2. $\mathrm{n}$ TOF NEAR station, experimental site. From LoI CERN-INTC-2020-073.

\section{The ${ }^{7} \mathrm{Be}(n, \alpha)$ reaction in the context of the Cosmological Lithium Problem}

The distribution of the Big-Bang Nucleosynthesis (BBN) abundances results by a well-established cosmological model describing the production of elements in the early stage of the Universe and it represents a cornerstone of the Big-Bang model. In fact, BBN reproduces observed primordial abundances for all isotopes except ${ }^{7} \mathrm{Li}$, for which it overestimates the relative abundance by approximately a factor three [3]. A possible explanation is related to the Nuclear Physics input of the nucleosynthesis network, describing the production and destruction rate of ${ }^{7} \mathrm{Be}$, being primordial ${ }^{7} \mathrm{Li}$ mostly produced by the decay of this isotope. In particular, while the main production channel, the ${ }^{3} \mathrm{He}(\alpha, \gamma)^{7} \mathrm{Be}$, is relatively well known, the reaction cross sections responsible for its destruction remain uncertain. Several measurements were performed to solve this problem [4-7]. They have shown that neither proton- nor deuteron-induced reactions can solve the Lithium problem. In this framework, neutron-induced reactions play a key role, in particular the ${ }^{7} \mathrm{Be}(n, p)^{7} \mathrm{Li}$, considered one of the main reaction for ${ }^{7} \mathrm{Be}$ destruction during $\mathrm{BBN}$. If ${ }^{7} \mathrm{Li}$ production is enhanced by neutron capture in the early stages of BBN $(T=50 \mathrm{keV})$ it can be rapidly burned by the ${ }^{7} \mathrm{Be}(p, \alpha)$ reaction. In 1988 , a measurement of the cross-section of the ${ }^{7} \mathrm{Be}(n, p)$ reaction from thermal to $13.5 \mathrm{keV}$ was performed at LANSCE (Los Alamos) [8] and more recently at $n \_$TOF in a wider energy range [9]: the experimental results exclude a significant impact of this reaction on the ${ }^{7} \mathrm{Li}$ problem [9].

With respect to the ${ }^{7} \mathrm{Be}(n, p)$ channel, the contribution of the ${ }^{7} \mathrm{Be}(n, \alpha)$ reaction to the depletion of ${ }^{7} \mathrm{Be}$ during $\mathrm{BBN}$ has been considered negligible, due to its much lower cross-section. However, this assumption was never experimentally verified. For this reason, a large uncertainty in BBN calculation has been typically assigned to this reaction and just a single $(n, \alpha)$ measurement at thermal energy has been reported [10], while various theoretical extrapolations in the $\mathrm{keV}$ neutron energy region yield completely different results [11]. Consequently, a first measurement on this reaction in the $20-100 \mathrm{keV}$ neutron energy range would finally shed light on the role of the ${ }^{7} \operatorname{Be}(n, \alpha)$ reaction in the Cosmological Lithium Problem. Due to the very important astrophysical motivations and the recent availability of new n-beam facilities (n_TOF-CERN, SARAF-Israel, ILL-France), this subject is being investigated by several research groups worldwide. The complementarity of the experimental approaches used in this scientific quest will ensure the reliability of the result.

\section{The ${ }^{7} \operatorname{Be}(n, \alpha)$ reaction: the advanced experimental approach at $n$ _TOF}

The main challenge in the measurement of the ${ }^{7} \mathrm{Be}(n, \alpha)$ reaction in the energy range of interest for $\mathrm{BBN}$ is strictly related to its low cross section. Thus, two major problems arise: the production and availability of a suitable amount of the short living ${ }^{7} \mathrm{Be}$ isotope for the target and the implementation of an experimental setup with a $4 \pi$ coverage for ejectile detection. In order to measure the ${ }^{7} \mathrm{Be}(n, \alpha)$ cross sections with good statistical accuracy, a target of a few micrograms of ${ }^{7} \mathrm{Be}$ is needed. In this contest the group at Paul-Scherrer-Institute (PSI), Zurich, has been able to extract and separate a relatively large amount of ${ }^{7} \mathrm{Be}$, up to $8 \mu \mathrm{g}$ [12], thus finally making possible this measurement. This isotope has been produced and made available within the wellestablished INFN-CERN-PSI collaboration. The ${ }^{7} \mathrm{Be}$ has been extracted from the water cooling of the SINQ spallation source, purified and deposited on a proper backing. Two target deposition methods have been developed so far: electrodeposition and micro-drop deposition-evaporation, both the targets are shown in fig. 3. An initial activity of $18 \mathrm{GBq}$ was measured for each target, accounting for a total ${ }^{7} \mathrm{Be}$ mass of $2.8 \mu \mathrm{g}$.

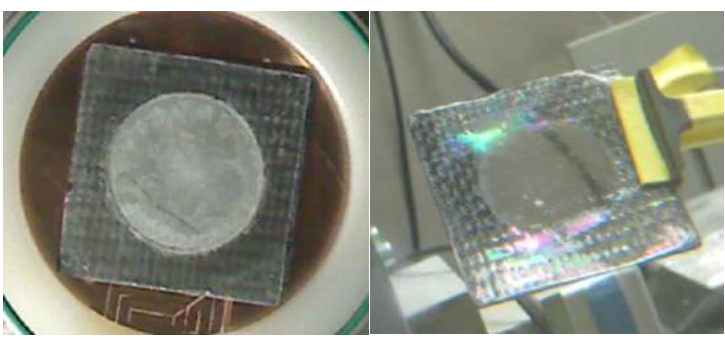

Fig. 3. ${ }^{7} \mathrm{Be}$ targets (PSI): electrodeposited target (left), evaporated target by micro-drop deposition (right). 
The two targets have been used in the setup, confirming a full consistency with respect to the target preparation. The main remaining challenge was to design and build an experimental setup, after suitable R\&D and in-beam test, able to sustain the large count rate related to the ${ }^{7} \mathrm{Be}$ radioactivity and minimize the background induced by the neutron beam. This was one of the major task of the proposed experiment, also important for future measurements of $(\mathrm{n}, \mathrm{cp})$ reactions at $\mathrm{n}$-TOF, as well as for nuclear technology, in particular for research related to fusion energy, and for Nuclear Medicine.

In the ${ }^{7} \mathrm{Be}(n, \alpha)^{4} \mathrm{He}$ reaction channel, the two alphaparticles are emitted back-to-back with a maximum energy of $9.5 \mathrm{MeV}$ (the $Q$-value of the reaction is 18.99 $\mathrm{MeV}$ ). The design of the setup exploited a double sandwich of Si-pad detector, with a ${ }^{7} \mathrm{Be}$ layer in between. The setup, placed directly in the neutron beam, is shown in fig 4 [13].

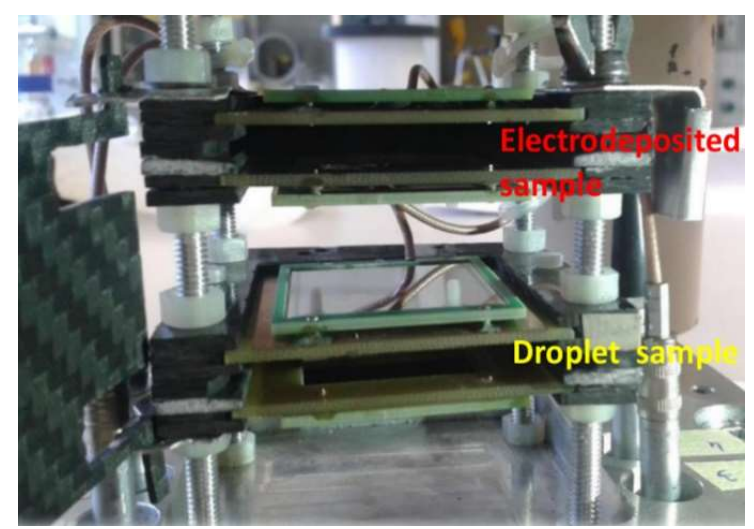

Fig. 4. Experimental setup. The two ${ }^{7} \mathrm{Be}$ targets have been inserted in between a couple of Si-pads, detecting alphaparticles in coincidence. All the system has been placed in line with the $n_{-}$TOF n-beam, for further details see ref. [13].

The geometrical configuration grants a quasi- $4 \pi$ coverage for the emitted alpha particles. Back-to-back coincidence is a crucial requirement for background suppression, while the double target configuration provided redundancy and mutual cross-check, suitable for revealing systematic errors. In this setup the measured ${ }^{7} \mathrm{Be}$ total target activity was $36 \mathrm{GBq}$, confirming that the radiation hardness of the $\mathrm{Si}$ detectors, in combination with the large $Q$-value of the reaction, leads to a very good discrimination of the coincidence events produced by the two alpha-particles. Such a performance was confirmed by the backgroundfree alpha-alpha correlation 2D-spectrum, as shown in fig. 4 .

At the same time we verified that the neutron induced background in the detectors, target contaminants, and target-backing are not affecting the measurement: in general competing reactions induced by the neutron beam on the detectors themselves, in particular $(n, p)$ and $(n, \gamma)$ reactions on $\mathrm{Si}$ (or C) and other material in the beam can pollute the data.

According to the existing predictions of the cross section, the measurement lasted several weeks. During the run, the performances of the setup were continuously monitored, confirming the excellent radiation hardness of the Si-pad, directly exposed to n-beam irradiation. Further details on the analysis and the results have been published in Physical Review Letters [14].

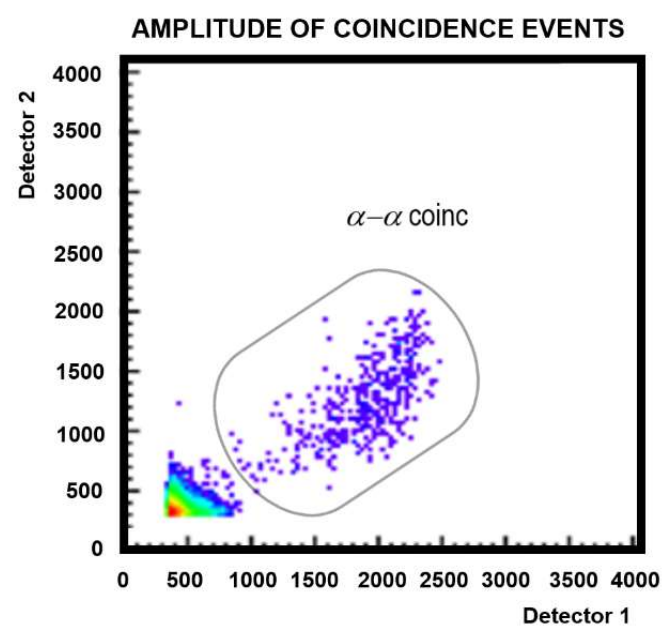

Fig. 5. Correlation plot, $\alpha-\alpha$ coincidences locus shows up fully separated with respect to the $\gamma$ and $n$-induced background on Silicon-pads and detector frame.

\section{Towards the measurement of the $n-n$ scattering length in EAR2}

The wide energy range covered by the $n$-beam provided by the $n_{-}$TOF facility makes the measurement of excitation function for few-body reactions involving observables relevant for fundamental Nuclear Physics possible. This is the case of the $n-n$ scattering length measurement, according to a LoI recently proposed and approved by the CERN INTC-committee (LoI CERNINTC-2020-051 / INTC-I-22021/09/202).

Due to the unavailability of free neutron targets, it has been realized, since five decades, that the neutronneutron $(n-n)$ cross section can be studied only through reactions involving two neutrons in the final state, mainly by the neutron-deuteron breakup $d(n, p) n n$.

A determination of the $n-n$ scattering length in a wide energy range (5-50 MeV) joined with a contextual $n-p$ scattering length measurement would represent a cornerstone for Nuclear Physics in investigating charge symmetry breaking and the nucleon-nucleon $(\mathrm{N}-\mathrm{N})$ force.

It must be mentioned that in 2006, the $\mathrm{n}$ TOF collaboration submitted a proposal (INTC-P-204) for such a measurement and the experiment was recommended for approval. Due to the demanding requirements in terms of detection efficiency and beam intensity it was not possible to carry out the measurement in the EAR1 station. Now, by the overall improvements of the facility, especially in terms of neutron flux and TOF resolution in the EAR2 station, the definition of the experimental setup can be finally approached in a realistic way.

The most challenging part of the experiment concerns the detection of the three outgoing particles of the $d(n, p) n n$ reaction in kinematic coincidence, thus 
leading to a complete three-body kinematic reconstruction. The demand of large efficiency in detecting two neutrons and one proton in coincidence, requires the development of new detection techniques. In this context the collaboration is developing a new deuterated active target for proton detection and an innovative system for neutron momentum reconstruction with high efficiency. Consequently, the first $\mathrm{n} \_$TOF deuterated active target «TaraT» has been proposed and preliminarly tested at NCSR DEMOKRITOS, Athens. The target design evolved from the proof-of-concept described in fig. 6 to the first prototype presented in fig. 7 .

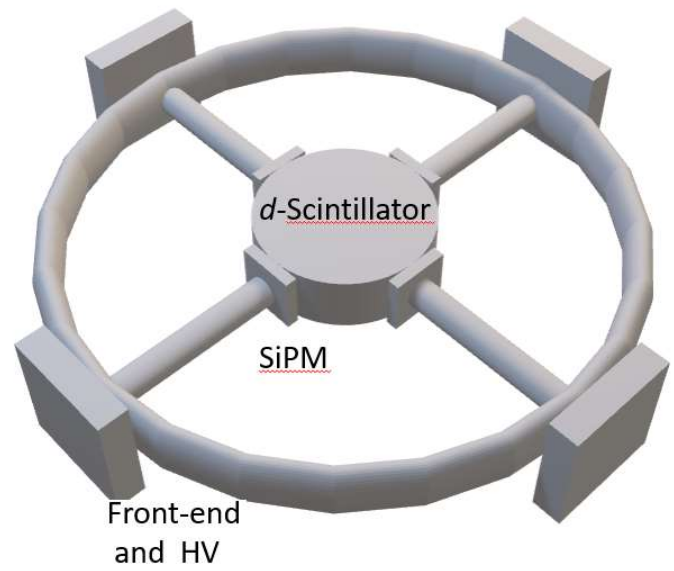

Fig. 6. The geometry of "TaraT", the Active Target.

The low-mass target assembly develops around the central deuterated crystal scintillator: in the first prototype shown in fig. 7 a $d$-stilbene crystal was kindly provided by the Lawrence Livermore National Laboratory (LLNL) [15]. The scintillation light is then collected by using four Silicon-Photomultiplier (SiPM) coupled to the side surface of the crystal. The external frame is supporting the front-end electronics (fastamplifiers and SiPM power supply). The cylindrical symmetry of the active target allows an easy rescaling of the deuterated scintillator in terms of diameter and shape as well as a fast maintenance and replacement of the activated parts.

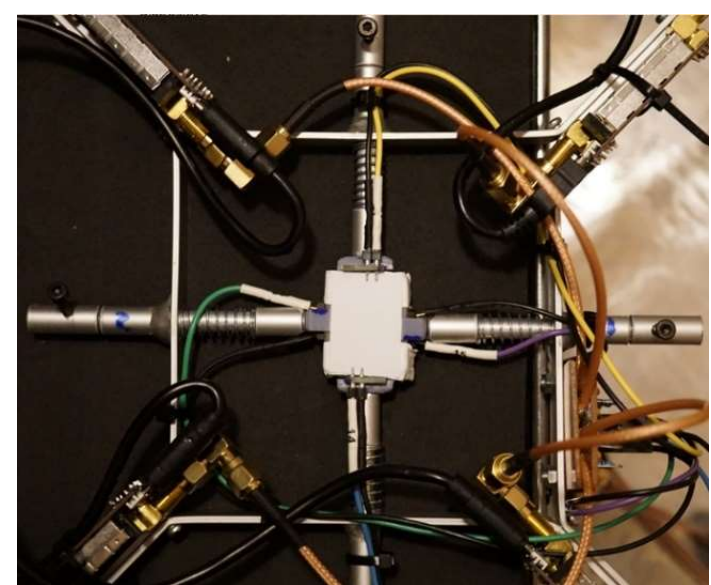

Fig. 7. The instrumented first "TaraT" prototype. The $d$ stilbene scintillator has been provided by LLNL.
The analysis of the n-beam test $\left(\mathrm{E}_{\mathrm{n}}=5-20 \mathrm{MeV}\right)$ performed at NCSR DEMOKRITOS confirmed a suitable dynamic range for the SiPM devices and a good time resolution (300 ps). The $n-\gamma$ Pulse-Shape Discrimination (PSD) capability of the stilbene scintillator was confirmed and a new statistical approach to the PSD is being implemented.

Finally, a test of the active target is planned in the $\mathrm{n}$ _TOF EAR2 station at CERN, after the commissioning of the new spallation target (i.e., at the end of 2021). This test will provide a crucial information on the $\gamma$-flash response of the setup. In fact, the strong electromagnetic pulse produced by the PS proton bunch impinging on the lead spallation target can couple to the detector and the front-end electronics, producing a shortening of the TOF dynamic range.

1. N. Colonna et al., Energy Environ. Sci. 3, 1910 (2010)

2. M. Sabaté-Gilarte et al., Eur. Phys. J. A 53, 210 (2017)

3. R.H. Cyburt et al., Rev. Mod. Phys. 88, 015004 (2016)

4. C. Angulo et al., Astrophys. J. 630, L105 (2005)

5. P. O’Malley et al., Phys. Rev. C 84, 042801(R) (2011)

6. O. S. Kirsebom and B. Davids, Phys. Rev. C 84, 058801 (2011)

7. C. Broggini, L. Canton, G. Fiorentini, and F. L. Villante, J. Cosmol. Astropart. Phys. 06 (2012) 30

8. P.E. Koehler et al, Phys. Rev. C 37, 917 (1988)

9. L. Damone et al., Phys. Rev. Lett. 121, 042701 (2018)

10. P. Bassi et al., Il Nuovo Cimento 28, 1049 (1963)

11. S. Q. Hou, J. J. He, S. Kubono, and Y. S. Chen, Phys. Rev. C 91, 055802 (2015)

12. E. Maugeri et al., Nucl. Instrum. Methods Phys. Res., Sect. A 889, 138 (2018)

13. L Cosentino et al., Nucl. Instrum. and Methods Phys. Res., Sect. A 830197 (2016)

14. M. Barbagallo et al., Phys. Rev. Lett. 117, 152701 (2016)

15. N.P. Zaitseva et al., Nucl. Instrum. and Methods Phys. Res. A 789, 8 (2015) 